\title{
Immune Effective Score As A Predictor Of Response To Neoadjuvant Trastuzumab Therapy And A Prognostic Indicator For HER2-positive Breast Cancer
}

\section{Xueying Wu}

PKU: Peking University

Chenyang Zhang

PKU: Peking University

Jing-wei Xiong

PKU: Peking University

Henghui Zhang ( $\square$ zhhbao@ccmu.edu.cn )

Capital Medical University Affiliated Beijing Ditan Hospital https://orcid.org/0000-0002-2820-7447

\section{Research Article}

Keywords: HER2-positive breast cancer, neoadjuvant, trastuzumab, pathological complete response, prognosis

Posted Date: March 18th, 2021

DOI: https://doi.org/10.21203/rs.3.rs-84422/v2

License: (c) (1) This work is licensed under a Creative Commons Attribution 4.0 International License. Read Full License

Version of Record: A version of this preprint was published at Current Oncology on January 10th, 2022. See the published version at https://doi.org/10.3390/curroncol29010026. 


\section{Abstract}

Background: HER2-positive breast cancer (BC) is a highly aggressive phenotype and accounts for 15$20 \%$ of all BC cases. The role of the host immune features in predictive response to anti-HER2 therapies and prognosis in $\mathrm{BC}$ is already suggested. We aimed to develop a predictive and prognostic model and examine its relevance to the clinical outcomes of patients with HER2-positive BC.

Methods: Based on the single-sample gene set enrichment analysis (sSGSEA) scores of the signatures related to neoadjuvant trastuzumab therapy response, an immune effective score (IES) was constructed using principal component analysis algorithms. A bioinformatic analysis using 4 independent cohorts (GSE66305, n=88; GSE130786, n=110; TCGA, n=123; METABRIC, $n=236$ ) established associations between IES and clinical outcomes.

Results: Genes associated with neoadjuvant trastuzumab therapy response enriched in pathways related to antitumor immune activities, including $T$ cell receptor signaling pathway, Natural killer cell-mediated cytotoxicity and NF-kappa B signaling pathway. IES was demonstrated to be a predictive biomarker to neoadjuvant trastuzumab therapy benefits (GSE66305: area under the curve (AUC) $=0.804$; GSE130786: AUC $=0.704$ ). In addition, IES was identified as an independent prognostic factor for overall survival (OS) in the TCGA cohort ( $P=0.036$, hazard ratio (HR): $0.66,95 \%$ confidence interval $(\mathrm{Cl}): 0.449-0.97)$ and METABRIC cohort $(P=0.037$, HR: $0.9,95 \% \mathrm{Cl}: 0.81-0.99)$.

Conclusions: The IES has a predictive value for response to neoadjuvant trastuzumab therapy and independent prognostic value for HER2-positive breast cancer.

\section{Background}

Breast cancer $(\mathrm{BC})$ with gene over-expression and/or amplification of Human Epidermal Growth Factor Receptor 2 (HER2) accounts for $15-20 \%$ of all BC cases ${ }^{[1]}$. Although HER2-positive BC is a highly aggressive phenotype, the emergence of trastuzumab and its application in the neoadjuvant therapy of early breast cancer has greatly enhanced the clinical outcomes of patients ${ }^{[2]}$. To date, the predictive biomarker for anti-HER2 agents is still HER2 overexpression or amplification. However, a number of such patients do not respond to HER2-targeted treatment in the neoadjuvant setting ${ }^{[3]}$. Potential predictive therapeutic biomarkers are therefore urgently needed to improve the efficacy of these therapies for HER2positive BC patients.

Currently, there is growing evidence that patients with high levels of immune infiltration may benefit more from the anti-HER2 monoclonal antibody trastuzumab ${ }^{[4,5]}$, indicating that tumor-infiltrating lymphocytes (TILs) might serve as a biomarker of anti-HER2 therapeutic response. On the other hand, HER2-positive BCs generally have higher TILs levels than Luminal A/B BCs, implying that HER2-positive BCs are usually more immunogenic ${ }^{[6,7]}$. Therefore, immune characteristics are important prognostic indicators for HER2positive $\mathrm{BCs}$ as demonstrated in several studies ${ }^{[8]}$. Collectively, these findings indicate an important role 
for immunity in the HER2-positive BC, both in the prediction of anti-HER2 therapeutic efficacy as well as the evaluation of prognosis. Nonetheless, the composition of the immune cell subsets is highly complex and it is still unclear which immune effectors are functional for clinical outcomes in HER2-positive BC.

In the present study, significant immune signatures associated with the therapeutic response to anti-HER2 were identified based on the weighted correlation network analysis (WGCNA) method. We then established an immune effective score (IES) of 557 tumors in four cohorts from patients with confirmed HER2-positive BC. We found IES to be a predictive factor in response to neoadjuvant trastuzumab therapy and an independent prognostic biomarker.

\section{Methods And Materials Data collection}

Single nucleotide variation (SNV) and copy number variation (CNV) data of TCGA-BRCA were obtained from the GDC Data Portal (https://portal.gdc.cancer.gov/). The RNAseq data and clinical information of TCGA-BRCA cohort were obtained from UCSC Xena (https://xena.ucsc.edu/). Microarray data were obtained from GEO (https://www.ncbi.nlm.nih.gov/geo/query/, GSE66305, GSE130786). Gene expression data and clinical information of METABRIC cohort were downloaded from the cBioPortal (http://www.cbioportal.org/). Patients enrolled in this study had confirmed positive (2+) immunohistochemistry HER-2 status, and were 557 in total (GSE66305, $n=88$; GSE130786, $n=110$; TCGA, $n=123$; METABRIC, $n=236$ ).

\section{Weighted gene co-expression network analysis}

The gene co-expression networks were constructed by the WGCNA package ${ }^{[9]}$ in R (version 3.6.3). A coexpression network was constructed by 5044 genes with variance ranked in the top $25 \%$, and the Pearson correlations were calculated between all genes. Based on the network's scale-free topology, a $\beta$ (softthresholding power) parameter was determined to reconstruct the network for strongly correlated genes and exclude weakly correlated genes.

\section{Immune-related signature analysis}

The gene sets for CD8 T cells, Tfh, Th1, NK, cytolytic activity, inflammation promoting and T cell costimulation were used in previous study ${ }^{[10]}$. Each signature score was calculated by single-sample gene set enrichment analysis (ssGSEA) using "GSVA" package (method = "ssgsea") in R (version 3.6.3).

\section{Gene set enrichment analysis (GSEA)}

Gene set enrichment analysis (GSEA) was performed using the GSEA software provided by the Broad Institute. Reactome pathway gene sets were obtained from MSigDB version 7.1 (http://software.broadinstitute.org/gsea/msigdb).

\section{Statistical analysis}


All statistical analyses were performed using R version 3.6.3 software (Institute for Statistics and Mathematics, Vienna, Austria; www.r-project.org). The Wilcoxon test was used to compare two continuous variables. Survival analysis was performed using a Kaplan-Meier survival plot, and the logrank test $P$-value was calculated. Statistical significance was set at $P<0.05$.

\section{Results}

\section{Genes associated with neoadjuvant trastuzumab therapy response are enriched in antitumor immune signaling pathways}

To identify genes related to neoadjuvant trastuzumab therapy response, we first constructed a coexpression network using samples from 88 patients with breast cancer who had undergone different neoadjuvant therapies (GSE66305). WGCNA was used with a screen out power from 1 to 20 , and $\beta=10$ was selected as the best value for the soft threshold of the network, resulting in a scale-free topology index $\left(R^{2}\right)$ of 0.9 and higher average connectivity (Fig. 1A). A total of 9 co-expression modules were constructed and are shown in different colors, and the module-trait heatmap is shown in Fig. 1B. The yellow, pink and red modules all had a high correlation with response to neoadjuvant trastuzumab therapy (all $R>0.35, P<0.001$ ), whereas for other therapies and modules no correlation was observed (Fig. 1B). We then performed enrichment analysis on the Kyoto Encyclopedia of Genes and Genomes (KEGG) to categorize the biological functions of genes found in these three modules using the "enrichKEGG" programming function in R's "clusterProfiler" package ${ }^{[11]}$. Interestingly, our data showed that genes associated with neoadjuvant trastuzumab therapy response were highly enriched in pathways closely related to antitumor immune activities, including the $T$ cell receptor signaling pathway, Natural killer cell-mediated cytotoxicity and NF-kappa B signaling pathway (Fig. 1C).

\section{Construction of immune effective score (IES)}

Previous research showed that cytotoxic $\mathrm{CD}^{+} \mathrm{T}$ cells $^{[12,13]}$, T helper type 1 (Th1) cells ${ }^{[14]}$ ), follicular helper $T(T f h)$ cells ${ }^{[15]}$ and Natural Killer (NK) cells ${ }^{[16]}$ were strongly associated with patient survival or response to therapy in BC. These reports, combined with our data, suggest that immune effective signatures may contribute to trastuzumab beneficial therapeutic effects. Therefore, seven signatures which have been reported for the classification of triple-negative breast cancers (TNBC) immune phenotypes ${ }^{[10]}$, including CD8 T cells, Tfh, Th1, NK, cytolytic activity, inflammation promoting and $T$ cell co-stimulation, were chosen for further analysis (see Table S1 for all gene sets). On the basis of the sSGSEA scores of the 7 signatures, a principal component analysis (PCA) was performed, and immune effective score (IES) of each patient was defined as the sum of the first two principal components. To test the accuracy of IES, we further calculated the tumor infiltrating immune cells (TILs) level by previously published method ${ }^{[17]}$, and examine the correlations of the IES and TILs. A statistically significant positive correlation between IES and infiltration of activated CD8 T cells (Spearman $R=0.88, P<0.001$; Fig. 2A), 
Th1 (Spearman $R=0.83, P<0.001$; Fig. 2B), Tfh (Spearman $R=0.77, P<0.001$; Fig. $2 \mathrm{C}$ ) and NK cells (Spearman $R=0.55, P<0.001$; Fig. 2D) was seen, suggesting IES could serve as a signature score, as expected.

\section{The IES predicts neoadjuvant trastuzumab therapy benefits}

Next, two datasets (GSE66305 and GSE130786) were used for investigating whether there was association between IES at baseline and pathological complete response (pCR) rates of neoadjuvant trastuzumab therapy. The first third IES value was was used as the cutoff for identifying a high-IES tumor. Results showed a higher pCR rate in the GSE66305 cohort high-IES community (50\% vs. $13 \%$; Fig. $2 \mathrm{E}$ ) and the GSE130786 cohort ( $64 \%$ vs. $40 \%$; Fig. 2G) compared with the corresponding low-IES groups. However, we did not observe a similar trend with other neoadjuvant therapies (Figure S1). Moreover, when the IES was evaluated as a continuous variable with the receiver-operating-characteristic (ROC) analyses, IES was also demonstrated to be a predictive biomarker of neoadjuvant trastuzumab therapy benefits (GSE66305: area under the curve (AUC) = 0.804; Fig. 2F; GSE130786: AUC =0.704; Fig. 2H).

\section{IES is associated with FGFR1 signaling in HER2-positive BC}

In addition to immune profiles, trastuzumab-resistance is also correlated with genomic changes such as mutations in PI3KCA ${ }^{[18]}, P T E N$ loss $^{[19]}$, and HER2 dimerization partners amplification ${ }^{[20]}$. Hence, we further utilized gene expression data, simple nucleotide variation (SNV), and copy number variation (CNV) information on HER2-positive BC patients in TCGA $(n=123)$ to identify biological processes linked to IES status. Patients in the TCGA cohort were classified into low and high (-IES) groups as the previously described, and GSEA analysis was carried out to identify the IES associated with biological signaling pathway. Since interferon- $y$ can be produced by the CD $8^{+}$T cells, Th1, Tfh and NK cells, it was no surprise that interferon-gamma signature genes were significantly enriched in high-IES samples (normalized enrichment scores (NES) $=2.4312$; Fig. 3A). However, it is worth noting that FGFR-PI3K signaling axis was enriched in the low-IES group (NES = - 1.5385; Fig. 3B; NES = - 1.5284; Fig. 3C). The mutational landscape of PI3K pathway-related genes showed that FGFR1 amplification was observed in only $2.5 \%$ (1/40) of high-IES samples, but 17.3\% (14/81) of low-IES samples (Fig. 3D). This implies that trastuzumab-resistance in low-IES patients may be related to activation of FGFR1 signaling pathway.

\section{Evaluation of IES for the prognostic prediction of HER2-positive BC in TCGA cohort and METABRIC cohort}

From a variety of studies, it is now well established that immune functions play an important prognostic role in $\mathrm{BC}^{[21-23]}$. Thus, we sought to explore whether the IES as a predictor in HER2-positive $\mathrm{BC}$ has prognostic potential. Clinical information data were collected from patients with confirmed HER2-positive status in the TCGA cohort $(n=123)$ and METABRIC cohort $(n=236)$. After multivariate Cox regression analysis of all relating factors, IES was identified as an independent prognostic factor for overall survival (OS) in the TCGA cohort ( $P=0.036$, hazard ratio (HR): $0.66,95 \%$ confidence interval (CI): 0.449-0.97; Fig. 4A) and METABRIC cohort ( $P=0.037$, HR: 0.9, 95\% Cl: 0.81-0.99; Figure S2A). As a grouping variable, there was a trend toward improvement in the OS for high-IES patients from TCGA cohort $(P=0.055$; 
Fig. 4B) and METABRIC cohort ( $P=0.065$; Figure S2B). Consistent with the OS results, IES $(\mathrm{P}=0.009$, HR: $0.61,95 \% \mathrm{Cl}: 0.42-0.88 ; \mathrm{Fig} .4 \mathrm{C}$ ) was also independently linked to progression-free interval (PFI) in the multivariate Cox regression analysis. Besides, patients assigned to the high-IES group had significantly better PFI than those who were assigned to the low-IES group ( $P=0.009$; Fig. 4D). In summary, our data suggest that the IES is independent of conventional clinical characteristics and performed well in survival prediction for HER2-positive BC.

\section{Discussion}

Previous studies have noted the strong relationship between the host immune response and neoadjuvant trastuzumab therapy of HER2-positive BC patients ${ }^{[4,5]}$. Researchers often use a single immune-related gene $^{[24]}$ or overall immune infiltration ${ }^{[8]}$ as a predictive biomarker for neoadjuvant trastuzumab therapy. Although these results have important implications, they may not systematically indicate an effector immune response in patients. In this study, we found that genes associated with response to neoadjuvant trastuzumab therapy were highly enriched in pathways closely related to antitumor immune activities. Interestingly, most of these pathways including cytotoxic CD8 + T cells, Th1, Tfh and NK signatures, were important in the production of IFN- $\gamma$. Hence, based on the above conclusion, we designed an IES to evaluate the immune effective phenotype. In two independent cohorts, high-IES patients showed a higher pCR rate for preoperative trastuzumab therapy than the low-IES cohort.

Several anti-HER2 agents have currently been used in the neoadjuvant setting, including trastuzumab, pertuzumab and lapatinib ${ }^{[25,26]}$. The combination of dual anti-HER2 blockade, such as trastuzumab plus lapatinib and trastuzumab plus pertuzumab, has shown increased effectiveness over single blockade in clinical studies (NeoALTTO trial, NCT00553358[27]; CHER-LOB trial, NCT00429299[28]; NeoSphere trial, NCT00545688 ${ }^{[29]}$. However, trastuzumab alone remains the standard of care in combination with systemic chemotherapy (paclitaxel-based), given the possibility of increased toxicity. A recent review showed a range of $\mathrm{pCR}$ rate between $29 \%$ and $52 \%$ for neoadjuvant trastuzumab therapy ${ }^{[2]}$. Due to the different chemotherapy regimens, as well as the inclusion/exclusion criteria, it is difficult to compare pCR levels between different studies. What is interesting about our data is the pCR rate from the GSE66305 cohort in the high-IES group (88 samples from CHER-LOB trial) reached $50 \%$, but the overall pCR rate in chemo + trastuzumab arm was only $25 \%$ in CHER-LOB trial ${ }^{[28]}$. In the GSE66305 cohort, patients from the high-IES group in chemo + trastuzumab arm also showed a higher pCR rate (64\%) than the average level previously reported. No similar results were observed in arms with other treatment measures, implying that IES may serve as a predictive biomarker specific to neoadjuvant trastuzumab therapy. In addition, based on TCGA cohort analysis we found an enrichment in the low-IES community for activation of the FGFR1-PI3K-mTOR signaling axis. FGFR1, as a member of trans-membrane receptor tyrosine kinase (RTKs), has been reported to be associated with resistance to anti-HER2 therapies ${ }^{[20,30]}$. These findings suggest that in combination with trastuzumab, patients in the low-IES group can derive more benefit from FGFR inhibitor therapies. However, FGFR inhibitors have not yet reached clinical routine practice in BC and therefore these results should be interpreted with caution. 
In addition, high rates of lymphocyte infiltration have been linked with a more favorable prognosis in patients with triple-negative and HER2-positive breast cancer ${ }^{[31]}$. This is an indication that the immune effective phenotype may also be associated with a better prognosis. We applied multivariate Cox regression analysis in TCGA cohort and METABRIC cohort to discern this property. Further, we demonstrated the significance of the IES in predicting survival in HER2-positive breast cancer. Besides, the PFI benefit was much more pronounced, indicating that patients with low-IES are prone to progression and recurrence.

\section{Limitations}

This study has several limitations. First, we did not have any survival information for the GEO cohorts to explore the prognostic value of IES for disease-free survival (DFS) and event-free survival (EFS) in this study. Second, there is a need for more validation cohorts to verify the prediction and prognostic validity of the IES. These limitations mean that the study findings should be interpreted with caution, and further studies are required.

\section{Conclusions}

Taken together, we constructed an IES which has predictive value for response to neoadjuvant trastuzumab therapy and independent prognostic value for HER2-positive breast cancer.

\section{Abbreviations}

AUC: area under the curve

BC: breast cancer

Cl: confidence interval

GEO: gene expression omnibus database

HR: hazard ratio

IES: immune effective score

KEGG: Kyoto Encyclopaedia of Genes and Genomes

NK: natural killer cells

OS: overall survival

PCA: principal component analysis 
pCR: pathological complete respons

PFI: progression-free interval

RD: residual disease

ROC: receiver operating characteristic

ssGSEA: single-sample gene set enrichment analysis

TCGA: the cancer genome atlas

Tfh: follicular helper T cells

Th1: T helper type 1 cells

TILs: tumor-infiltrating lymphocytes

TNBC: triple-negative breast cancer

WGCNA: weighted correlation network analysis

\section{Declarations}

\section{Ethics approval and consent to participate}

Not applicable. All analyses were based on previously published studies.

\section{Consent for publication}

Not applicable.

\section{Availability of data and materials}

The datasets generated and/or analysed during the current study are available in the GDC Data Portal (https://portal.gdc.cancer.gov/), UCSC Xena (https://xena.ucsc.edu/), GEO database (https://www.ncbi.nlm.nih.gov/geo/query/), and cBioPortal (http://www.cbioportal.org/).

\section{Competing interests}

The authors declare that they have no competing interests. 


\section{Funding}

This work was supported by the National Key Sci-Tech Special Project of China (NO. 2018ZX10302207) and the National Natural Science Foundation of China (NO. 81774234). The funders did not play any role in the design of the study, the decision to publish or the manuscript preparation.

\section{Authors' contributions}

H.Z. and J.X. conceived and designed this study; C.Z. participated in the data arrangement; X.W. analyzed the data and interpreted the results; X.W. drafted the manuscript; All authors read and approved the final version of the manuscript.

\section{Acknowledgments}

First, the authors gratefully acknowledge the TCGA project organizers and GEO data repository for the public datasets. Secondly, we thank the METABRIC team for freely making their data available for analysis.

\section{References}

1. LoibI S, Gianni L. HER2-positive breast cancer. Lancet. 2017;389:2415-29.

2. Brandao M, et al. Combination therapies for the treatment of HER2-positive breast cancer: current and future prospects. Expert Rev Anticancer Ther. 2018;18:629-49.

3. Cameron D, et al. 11 years' follow-up of trastuzumab after adjuvant chemotherapy in HER2-positive early breast cancer: final analysis of the HERceptin Adjuvant (HERA) trial. Lancet. 2017;389:1195205.

4. Ingold Heppner B, et al. Tumor-Infiltrating Lymphocytes: A Predictive and Prognostic Biomarker in Neoadjuvant-Treated HER2-Positive Breast Cancer. Clin Cancer Res. 2016;22:5747-54.

5. Loi S, et al. Tumor infiltrating lymphocytes are prognostic in triple negative breast cancer and predictive for trastuzumab benefit in early breast cancer: results from the FinHER trial. Ann Oncol. 2014;25:1544-50.

6. Loi S, et al. Prognostic and predictive value of tumor-infiltrating lymphocytes in a phase III randomized adjuvant breast cancer trial in node-positive breast cancer comparing the addition of docetaxel to doxorubicin with doxorubicin-based chemotherapy: BIG 02-98. J Clin Oncol. 2013;31:860-7.

7. Stanton SE, Adams S, Disis ML. Variation in the Incidence and Magnitude of Tumor-Infiltrating Lymphocytes in Breast Cancer Subtypes: A Systematic Review. JAMA Oncol. 2016;2:1354-60. 
8. Denkert C, et al. Tumour-infiltrating lymphocytes and prognosis in different subtypes of breast cancer: a pooled analysis of 3771 patients treated with neoadjuvant therapy. Lancet Oncol. 2018;19:40-50.

9. Langfelder P, Horvath S. WGCNA: an R package for weighted correlation network analysis. BMC Bioinformatics. 2008;9:559.

10. He Y, et al. Classification of triple-negative breast cancers based on Immunogenomic profiling. J Exp Clin Cancer Res. 2018;37:327.

11. Yu G, et al. clusterProfiler: an R package for comparing biological themes among gene clusters. OMICS. 2012;16:284-7.

12. Mahmoud SM, et al. Tumor-infiltrating CD8 + lymphocytes predict clinical outcome in breast cancer. J Clin Oncol. 2011;29:1949-55.

13. Seo AN, et al. Tumour-infiltrating CD8 + lymphocytes as an independent predictive factor for pathological complete response to primary systemic therapy in breast cancer. $\mathrm{Br} \mathrm{J}$ Cancer. 2013;109:2705-13.

14. Costa RLB, Soliman H, Czerniecki BJ. The clinical development of vaccines for HER2(+) breast cancer: Current landscape and future perspectives. Cancer Treat Rev. 2017;61:107-15.

15. Gu-Trantien C, et al. CD4(+) follicular helper T cell infiltration predicts breast cancer survival. J Clin Invest. 2013;123:2873-92.

16. Tian X, et al. Herceptin Enhances the Antitumor Effect of Natural Killer Cells on Breast Cancer Cells Expressing Human Epidermal Growth Factor Receptor-2. Front Immunol. 2017;8:1426.

17. Charoentong P, et al. Pan-cancer Immunogenomic Analyses Reveal Genotype-Immunophenotype Relationships and Predictors of Response to Checkpoint Blockade. Cell Rep. 2017;18:248-62.

18. Kataoka Y, et al. Association between gain-of-function mutations in PIK3CA and resistance to HER2targeted agents in HER2-amplified breast cancer cell lines. Ann Oncol. 2010;21:255-62.

19. Nagata $Y$, et al. PTEN activation contributes to tumor inhibition by trastuzumab, and loss of PTEN predicts trastuzumab resistance in patients. Cancer Cell. 2004;6:117-27.

20. Hanker AB, et al. HER2-Overexpressing Breast Cancers Amplify FGFR Signaling upon Acquisition of Resistance to Dual Therapeutic Blockade of HER2. Clin Cancer Res. 2017;23:4323-34.

21. Chen $X Y$, et al. Prognostic role of immune infiltrates in breast ductal carcinoma in situ. Breast Cancer Res Treat. 2019;177:17-27.

22. Toss MS, et al. The prognostic significance of immune microenvironment in breast ductal carcinoma in situ. Br J Cancer. 2020;122:1496-506.

23. Liu F, et al. CD8(+) cytotoxic T cell and FOXP3(+) regulatory $T$ cell infiltration in relation to breast cancer survival and molecular subtypes. Breast Cancer Res Treat. 2011;130:645-55.

24. Varadan V, et al. Immune Signatures Following Single Dose Trastuzumab Predict Pathologic Response to PreoperativeTrastuzumab and Chemotherapy in HER2-Positive Early Breast Cancer. Clin Cancer Res. 2016;22:3249-59. 
25. Gradishar WJ, et al, Cancer B. Version 3.2020, NCCN Clinical Practice Guidelines in Oncology. J Natl Compr Canc Netw. 2020;18:452-78.

26. Li Q, et al., CSCO breast cancer guideline: precise, economical and oriental. Sci China Life Sci. 2020.

27. Huober J, et al. Survival outcomes of the NeoALTTO study (BIG 1-06): updated results of a randomised multicenter phase III neoadjuvant clinical trial in patients with HER2-positive primary breast cancer. Eur J Cancer. 2019;118:169-77.

28. Guarneri V, et al. Preoperative chemotherapy plus trastuzumab, lapatinib, or both in human epidermal growth factor receptor 2-positive operable breast cancer: results of the randomized phase II CHERLOB study. J Clin Oncol. 2012;30:1989-95.

29. Gianni L, et al. Efficacy and safety of neoadjuvant pertuzumab and trastuzumab in women with locally advanced, inflammatory, or early HER2-positive breast cancer (NeoSphere): a randomised multicentre, open-label, phase 2 trial. Lancet Oncol. 2012;13:25-32.

30. Vernieri $\mathrm{C}$, et al. Resistance mechanisms to anti-HER2 therapies in HER2-positive breast cancer: Current knowledge, new research directions and therapeutic perspectives. Crit Rev Oncol Hematol. 2019;139:53-66.

31. Savas P, et al. Clinical relevance of host immunity in breast cancer: from TILs to the clinic. Nat Rev Clin Oncol. 2016;13:228-41.

\section{Figures}



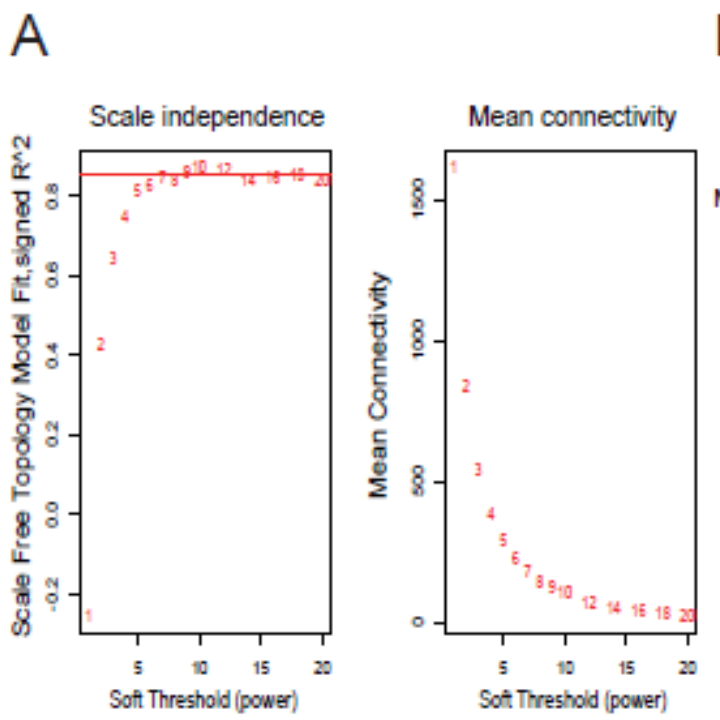

B

\section{Module-trait relationships}
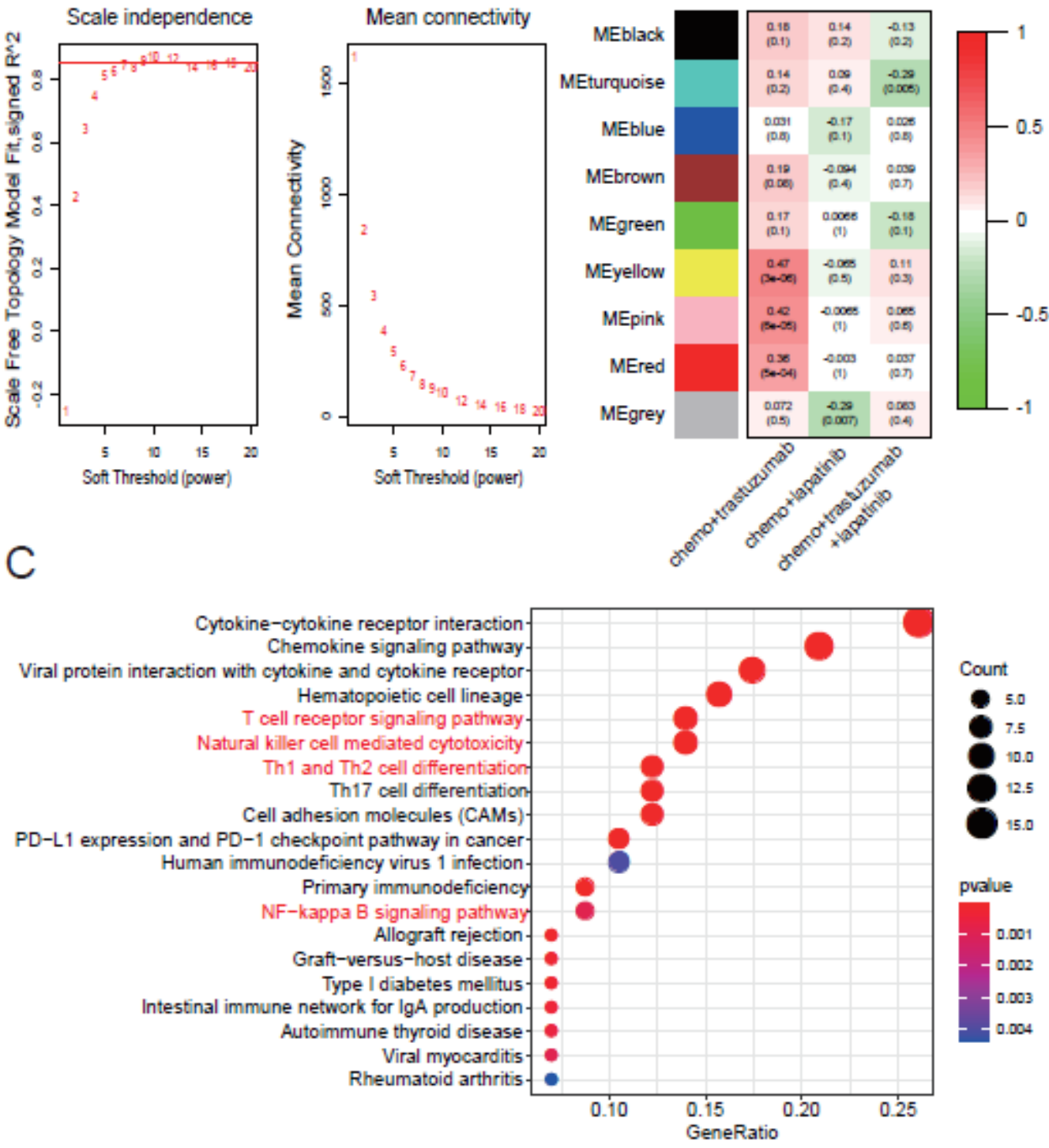

\section{Figure 1}

Construction of the gene co-expression network from patients exposed to neoadjuvant anti-HER2 therapies. (A) Selection of the soft-thresholding powers. The left panel shows the scale-free fit index versus the soft-thresholding power. The right panel displays the mean connectivity versus softthresholding power. (B) Module-trait relationships. Each row represents a ME, the three columns represent the response to chemo+trastuzumab, chemo+lapatinib, and chemo+trastuzumab+lapatinib, respectively, and each cell contains the corresponding correlation and P-value. The matrix is color-coded by correlation according to the color legend. Chemo: chemotherapy. (C) KEGG enrichment analysis of co-expression modules identified by WGCNA. KEGG: the Kyoto Encyclopaedia of Genes and Genomes. The y-axis represents the number of gene counts in the KEGG term. The intensity and color of dots are indicated on the right side of the heatmap and are represented by their corresponding P-values. 

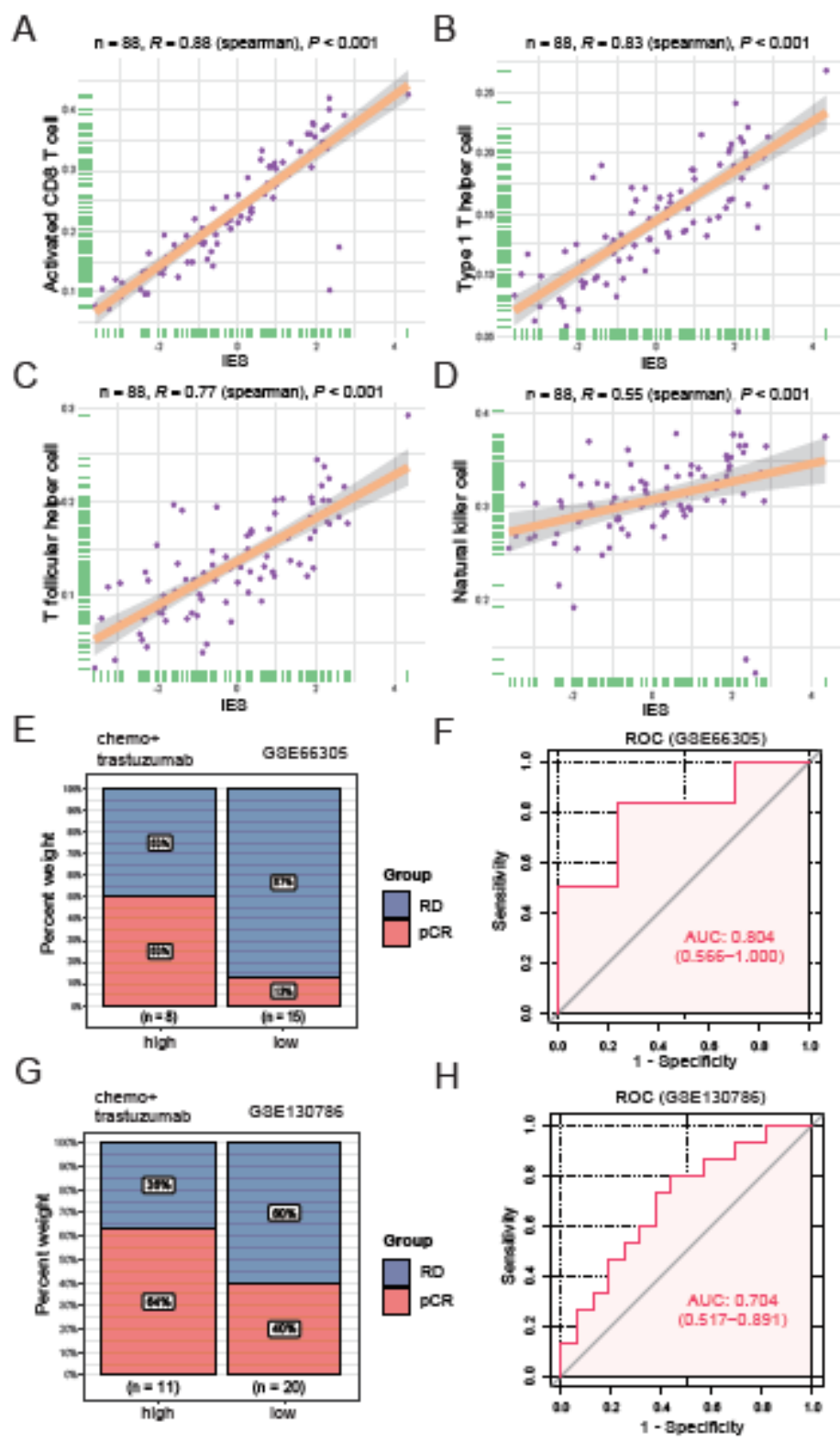

Figure 2

Functional and predictive analysis of immune effective score (IES). Scatter plots display the positive correlation between IES and activated CD8 T cell (A), type $1 \mathrm{~T}$ helper cell (B), T follicular helper cell (C), natural killer cell (D). The correlation coefficient $(R)$ and $P$ values were calculated by Spearman's rank correlation test are given in the figure. Bar plots depict the clinical response rate to neoadjuvant trastuzumab therapy in high or low IES groups in the GSE66305 cohort (E) and GSE130786 cohort (G). pCR: complete response; RD: residual disease. Receiver operating characteristic (ROC) analysis for predicting PCR to neoadjuvant trastuzumab therapy in the GSE66305 cohort (F) and GSE130786 cohort $(\mathrm{H})$. Area under the curve (AUC) values and $95 \%$ confidence interval $(\mathrm{Cl})$ are presented. 


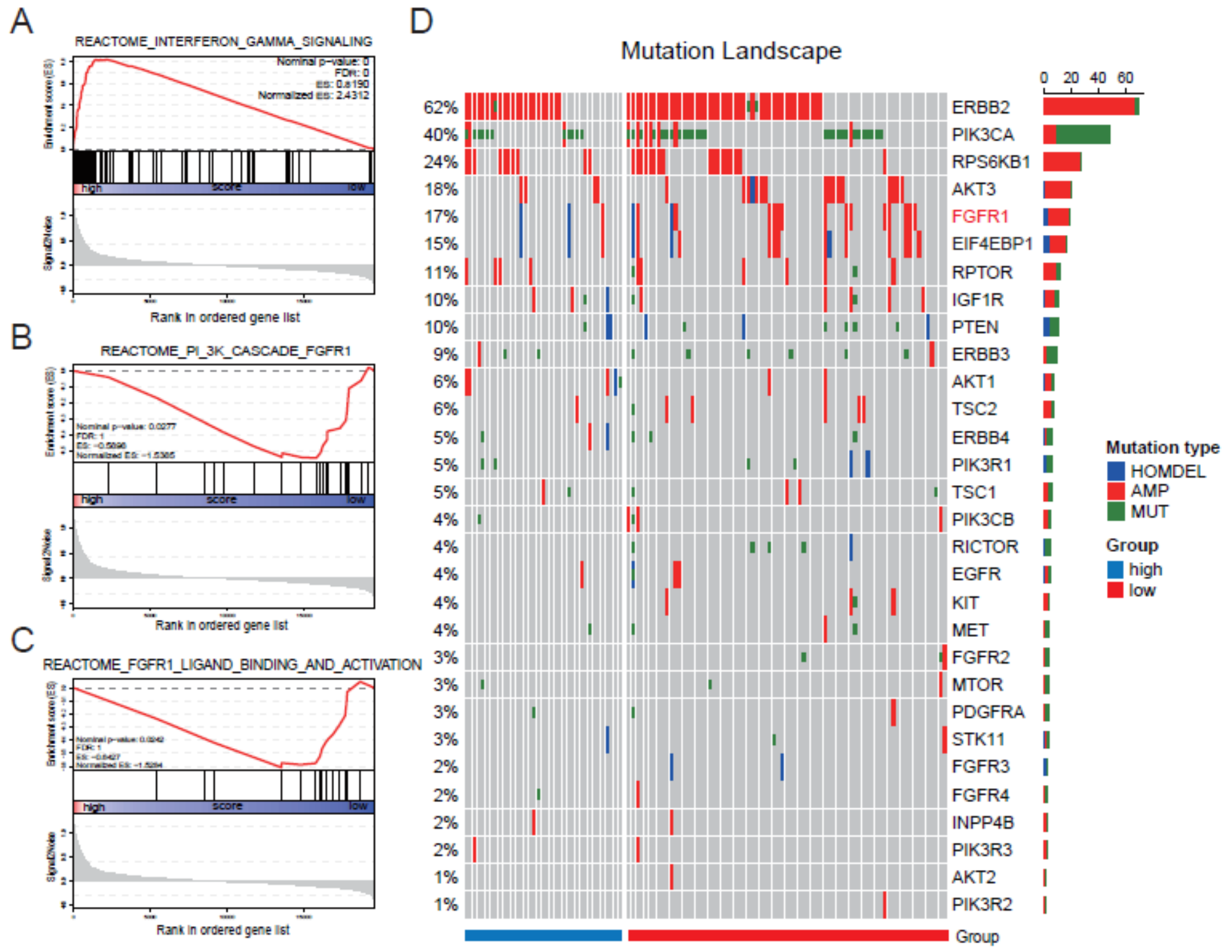

\section{Figure 3}

Low-IES status is associated with FGFR1 signaling. Enrichment plots from gene set enrichment analysis (GSEA) for the interferon-gamma signaling (A), PI3K cascade FGFR1 signaling (B) and FGFR1 ligand binding and activation signaling (C). Normalized enrichment scores (NES) and P values are also shown. Mutational landscape of PI3K pathway-related genes in the TCGA cohort (D). The column and row represent patients and genes, respectively. The patients are displayed in a descending order based on the number of mutated genes. The right panel indicates the frequency of gene mutations. Different colors indicate different types of mutations. Gray denotes no mutations. HOMDEL: homozygous deletion; AMP: amplification; MUT: mutation. 
A

Hazard ratio

B
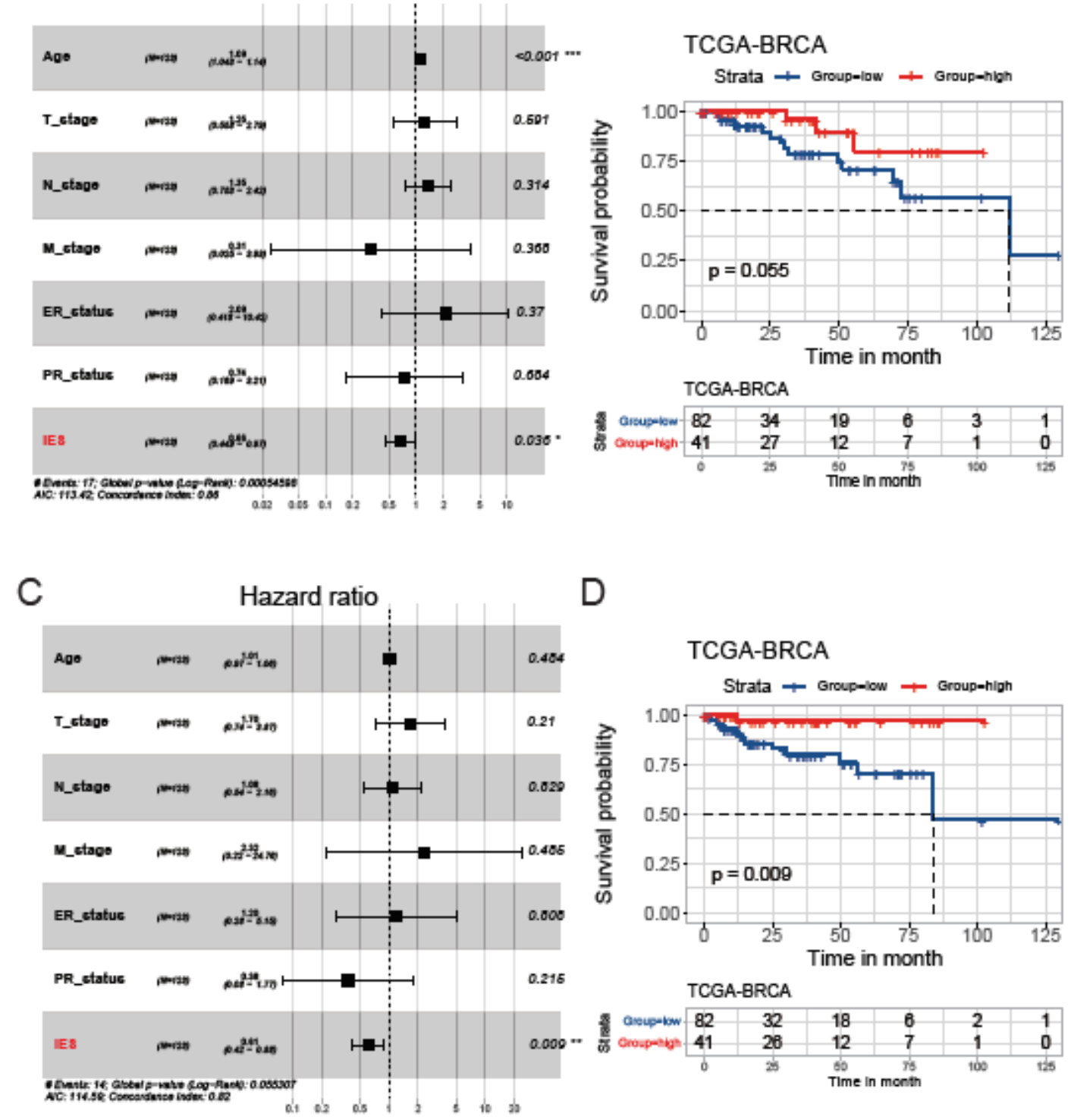

$\mathrm{D}$

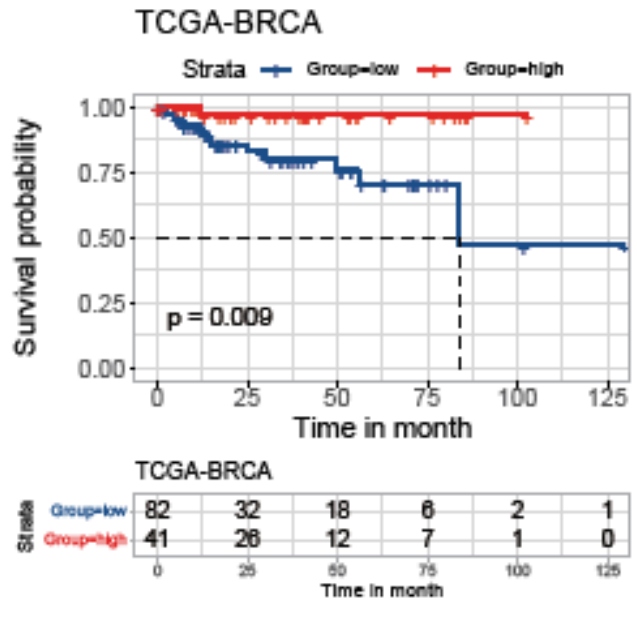

Figure 4

Prognostic analysis of the IES in the TCGA cohort. Forest plots of multivariate Cox regression proportional hazards regression analysis of OS (A) and PFI (C) in the TCGA cohort. P value was calculated by cox regression ( $\mathrm{P}<0.05,{ }^{\star} \mathrm{P}<0.01$, $\star \star \star * P<0.001$ ). OS: overall survival; PFI: progressionfree interval. Kaplan-Meier survival curves showed the OS (B) and PFI (D) stratified by low/high-IES in the TCGA cohort. $P$ values were obtained by the log-rank test.

\section{Supplementary Files}

This is a list of supplementary files associated with this preprint. Click to download.

- TableS1.docx

- figureS1.pdf 
- figureS2.pdf

Page 16/16 